\title{
Reconcile muon g-2 anomaly with LHC data in SUGRA with generalized gravity mediation
}

\author{
Fei Wang, ${ }^{a, b}$ Wenyu Wang $^{c}$ and Jin Min Yang ${ }^{b}$ \\ ${ }^{a}$ Department of Physics and Engineering, Zhengzhou University, \\ Zhengzhou 450000, P.R. China \\ ${ }^{b}$ State Key Laboratory of Theoretical Physics, Institute of Theoretical Physics, \\ Chinese Academy of Sciences, \\ Beijing 100190, P.R. China \\ ${ }^{c}$ Institute of Theoretical Physics, College of Applied Science, Beijing University of Technology, \\ Beijing 100124, P. R. China \\ E-mail: feiwang@zzu.edu.cn, wywang@mail.itp.ac.cn, jmyang@itp.ac.cn
}

\begin{abstract}
From generalized gravity mediation we build a SUGRA scenario in which the gluino is much heavier than the electroweak gauginos at the GUT scale. We find that such a non-universal gaugino scenario with very heavy gluino at the GUT scale can be naturally obtained with proper high dimensional operators in the framework of SU(5) GUT. Then, due to the effects of heavy gluino, at the weak scale all colored sparticles are heavy while the uncolored sparticles are light, which can explain the Brookhaven muon $g-2$ measurement while satisfying the collider constraints (both the $125 \mathrm{GeV}$ Higgs mass and the direct search limits of sparticles) and dark matter requirements. We also find that, in order to explain the muon $g-2$ measurement, the neutralino dark matter is lighter than $200 \mathrm{GeV}$ in our scenario, which can be mostly covered by the future Xenon1T experiment.
\end{abstract}

KeYWORDS: Supersymmetry Phenomenology

ArXiv EPRINT: 1504.00505 


\section{Contents}

1 Introduction 1

2 SUGRA with heavy-gluino constructed from generalized gravity mediation

3 Phenomenological constraints $\quad 6$

$\begin{array}{lll}4 \text { Conclusion } & 10\end{array}$

\section{Introduction}

If the particle discovered by the ATLAS and CMS collaborations of the LHC $[1,2]$ is indeed the long missing standard model (SM) Higgs boson, then the hierarchy problem related to the fundamental scalar may readily indicate some new physics beyond the SM. Another hint of new physics arises from the precise measurement of the muon anomalous magnetic moment by the Brookhaven experiment $[3,4]$, which gives a larger value than the SM prediction and the discrepancy is about $3 \sigma[5-8]$.

Among the new physics theories, the low energy supersymmetry (SUSY), which was initially proposed to solve the gauge hierarchy problem, is still regarded as one of the most appealing extensions. The observed $125 \mathrm{GeV}$ Higgs boson at the LHC falls miraculously within the narrow $115-135 \mathrm{GeV}$ "window" predicted by the minimal supersymmetric standard model (MSSM). Besides, in the framework of low energy SUSY, the three gauge couplings can naturally be unified [9-14], the cosmic cold dark matter can be naturally explained, the vacuum instability problem can be solved, and the muon $g-2$ discrepancy can also be accounted.

However, low energy SUSY also encounter some difficulties in the LHC era. The heavy top-squarks needed by a $125 \mathrm{GeV}$ Higgs boson, ${ }^{1}$ the null search results of sparticles and the perfect agreement of $B_{s}^{0} \rightarrow \mu^{+} \mu^{-}$with the SM prediction all imply SUSY at a rather high scale. Actually, the LHC data has already pushed the gluino and squarks of first two generations to $\mathrm{TeV}$ scale [17-20], i.e., $m_{\tilde{g}}>1.5 \mathrm{TeV}$ for $m_{\tilde{q}} \sim m_{\tilde{g}}$ and $m_{\tilde{g}} \gtrsim 1 \mathrm{TeV}$ for $m_{\tilde{q}} \gg m_{\tilde{g}}$, while for top-squarks the bounds from the LHC search are model-dependent, e.g., above $600 \mathrm{GeV}$ in natural SUSY [21]. On the other hand, if the muon $g_{\mu}-2$ anomaly is solved in the framework of SUSY, the relevent electroweak sparticles (smuons, neutralinos and charginos) need to be around $\mathcal{O}(100) \mathrm{GeV}$ for a $\tan \beta$ value of order $\mathcal{O}(10)$. So it seems that low energy SUSY should be realized in a more involved way [22-26].

\footnotetext{
${ }^{1}$ Note that in the non-minimal SUSY models like the next-to-minimal SUSY model such heavy topsquarks are not needed due to the additional tree-level contributions to the Higgs mass (for a comparative study of different SUSY models confronted with the LHC Higgs data, see, e.g., [15, 16]).
} 
If SUSY is required to account for the muon $g-2$ anomaly without contradiction with the recent LHC results, a split spectrum for spartilces is favored, which has one scale (relatively high) governing the colored sparticle masses and the other scale (relatively low) governing the uncolored sparticle masses [27, 28]. This can be realized in a supergravity (SUGRA) grand unified model called gluino-SUGRA [29] which has non-universal gaugino masses [30-36], with the gluino being much heavier than the electroweak gauginos at the GUT scale [29].

In this note we try to build such a gluino-SUGRA model from the generalized gravity mediation of SUSY breaking [37-40]. Oue results show that this scenario can be naturally obtained with proper high dimensional operators in the framework of SU(5) GUT. Then, due to the effects of heavy gluino, at the weak scale all colored sparticles are heavy while the uncolored spartilces are light, which can explain the Brookhaven muon $g-2$ measurement while satisfying the collider constraints and dark matter requirements. We also find that, in order to explain the muon $g-2$ measurement, the neutralino dark matter is below $200 \mathrm{GeV}$ in this scenario, which can be mostly covered by the future Xenon1T experiment.

This paper is organized as follows. In section 2, we construct a gluino-SUGRA model in the framework of SU(5) GUT from the generalized gravity mediation. In section 3, we examine the phenomenological constraints on our scenario, which are from the muon $g-2$, the LHC data, and the dark matter relic density and direct detection limits. Section 4 contains our conclusions.

\section{SUGRA with heavy-gluino constructed from generalized gravity me- diation}

To mediate the SUSY breaking effects from the hidden sector to the visible sector, many types of mechanisms are proposed, for example, gravity mediation [41-49], gauge mediation [50-56] and anomaly mediation [57, 58]. Among these mechanisms, the gravity mediation is a very predictive scenario. In this scenario the SM-like Higgs boson mass lies close to the upper limit $130 \mathrm{GeV}$ predicted in grand unified SUGRA models [59, 60].

In the popular gravity mediation scenario, the Kähler potential is assumed to be minimal. When certain high-representation chiral fields of the GUT group are involved in the nonrenormalizable Kähler potential, the kinetic terms of superfields can have alternative contributions after the GUT symmetry breaking. New nonrenormalizable terms in the superpotential involving high-representation fields can also be important. In general, both gauge singlet and non-singlet can acquire non-vanishing F-term VEVs to break supersymmetry. We will focus on the SU(5) grand unified SUGRA model in our analysis.

A general form of the kinetic terms for vector supermulitplet is

$$
\mathcal{L} \supseteq \int d^{2} \theta \tau\left(W^{a} W^{a}+a_{1} \frac{S}{M_{*}} W^{a} W^{a}+b_{1} \frac{\Phi_{a b}}{M_{*}} W^{a} W^{b}\right)
$$

with ' $\Phi$ ' denoting a GUT group non-singlet chiral supermultiplet and ' $S^{\prime}$ a GUT group singlet which can acquire a VEV of order (or below) $M_{*}$. 
From the symmetric product of SU(5) adjoint

$$
(24 \otimes 24)_{\text {symmetric }}=1 \oplus \mathbf{2 4} \oplus \mathbf{7 5} \oplus \mathbf{2 0 0},
$$

we can see that the non-renormalizable terms can be constructed with $\mathbf{2 4 , 7 5 , 2 0 0}$ representation chiral supermultiplets of SU(5). For simplicity, we assume that only $\mathbf{7 5}$ representation chiral field appears in eq. (2.1) and in the Kahler potential of the form

$$
K=\phi^{\dagger} \phi+\frac{c_{1}}{M_{*}} \sum_{r} \phi_{a}^{\dagger} S \phi_{a}+\frac{c_{1}^{\prime}}{M_{*}^{2}} \sum_{r} \phi_{a}^{\dagger}\left(\bar{\Phi}^{\mathbf{7 5}} \otimes \Phi^{75}\right)_{a b}^{r} \phi_{b},
$$

with $r$ denoting some representation from production expansion of $\mathbf{7 5} \otimes \mathbf{7 5}$. We assume that the superfield $\Phi_{75}$ acquires both the lowest component and F-term VEVs. After the GUT singlet $S$ field and $\mathbf{7 5}$ field acquire the lowest component VEVs

$$
<\Phi_{75}>_{a b}=v_{75} U_{a b},
$$

with the universal group factor $U_{a b}$ given in terms of $10 \times 10$ matrix as

$$
U_{a b}=\frac{1}{\sqrt{12}}(1,1,1,-1,-1,-1,-1,-1,-1,3),
$$

the wave-function normalization factor for the gauge kinetic term will have the form

$$
Z_{i}=1+a_{1} \frac{<S>}{M_{*}}+b_{1} \frac{<\Phi>_{i}}{M_{*}} \equiv \alpha+\beta_{i}
$$

with the ratios of the non-universal parts given by $\beta_{1}: \beta_{2}: \beta_{3}=-5: 3: 1$.

The F-term VEV of $\Phi^{75}$ given by $\left(F_{\Phi}\right)_{a b}=F_{\Phi} \cdot U_{a b}$ will lead to a non-canonical gaugino mass ratio

$$
M_{1}: M_{2}: M_{3}=-b_{1} \frac{5}{4 \sqrt{3}} \frac{F_{\mathbf{7 5}}}{M_{*}}: b_{1} \frac{3}{4 \sqrt{3}} \frac{F_{\mathbf{7 5}}}{M_{*}}: b_{1} \frac{1}{4 \sqrt{3}} \frac{F_{\mathbf{7 5}}}{M_{*}} .
$$

which, after re-scaling the normalization factor, will give a physical non-universal gaugino mass ratio

$$
M_{1}: M_{2}: M_{3}=\frac{-5}{Z_{1}}: \frac{3}{Z_{2}}: \frac{1}{Z_{3}} .
$$

For the choice of coefficient $Z_{3} \equiv \alpha+\beta_{3} \approx \mathcal{O}(0.1) \approx 0$, we can fix the value of $\alpha$ and thus the value of $Z_{1}, Z_{2}$ correspondingly. The ratio for $Z_{i}$ will be given approximately by

$$
Z_{1}: Z_{2}: Z_{3} \approx-6: 2: Z_{3},
$$

so we can obtain

$$
M_{1}: M_{2}: M_{3}=\frac{5}{6}: \frac{3}{2}: \frac{1}{Z_{3}} .
$$

We can see that at the GUT scale the gluino can be much heavier than bino and wino. On the other hand, the gluino will in general not be too heavier than the other two if no fine-tuning in the normalization factor is introduced. 
From group theory we know

$$
75 \otimes 75 \supset 1 \oplus \mathbf{2 4} \oplus \mathbf{7 5} \oplus \mathbf{2 0 0},
$$

so a unnormalized universal sfermion mass can be generated from Kahler potential by F-term VEVs of $\mathbf{7 5}$ :

$$
m_{\tilde{10}_{i}, \tilde{\overline{5}}_{i}, \tilde{H}_{u, d}}^{2}=\frac{3}{2} c_{\mathbf{1}}^{\prime} \frac{\left|F_{\mathbf{7 5}}\right|^{2}}{M_{*}^{2}}
$$

Note that there are many possible contractions of group factors in the Kahler potential and we adopt here the simplest case with $\left(\bar{\Phi}_{\mathbf{7 5}} \otimes \Phi_{\mathbf{7 5}}\right)_{a b} \propto \delta_{a b}$. On the other hand, it can be seen from formula (2.3) that the kinetic terms for matter contents will also get additional contributions from the GUT breaking effects by the lowest component VEVs of $\mathbf{7 5}$. So the unnormalized universal sfermion masses should be rescaled with respect to the kinetic factor to get the physical soft masses

$$
m_{\tilde{10}_{i}, \tilde{\overline{5}}_{i}, \tilde{H}_{u, d}}^{2}=\frac{3}{2 Z_{\phi}} c_{1}^{\prime} \frac{\left|F_{\mathbf{7 5}}\right|^{2}}{M_{*}^{2}} .
$$

with possible kinetic factor $Z_{\phi}$ as

$$
Z_{\phi}=1+\frac{c_{1}\langle S\rangle}{M_{*}}+c_{1}^{\prime} \frac{v_{75}^{2}}{M_{*}^{2}} \approx 1+\frac{c_{1}\langle S\rangle}{M_{*}} \equiv Z_{U}
$$

Therefore, the universal sfermion mass can be set as an free parameter in our scenario. The universal sfermion masses, which control the masses for slepton, should not be heavy in order to explain the $g_{\mu}-2$ anomaly. The squarks, on the other hand, will receive large corrections from gluino loops. So the typical universal sfermion mass scale should not be too larger than that of the lightest gaugino.

The trilinear term can be generated from the non-renormalization operators in the superpotential involving $\mathbf{7 5}$ superfield

$$
W \supset\left(\frac{\Phi_{a b}^{75}}{M_{*}}+c_{1} \frac{S \Phi_{a b}^{75}}{M_{*}^{2}}\right) \sum_{i, j=1}^{3}\left(y_{i j} \mathbf{1 0} i \otimes \mathbf{1 0}_{j} \otimes \mathbf{5}_{H_{u}}+y_{i j}^{\prime} \mathbf{1 0} \mathbf{0}_{i} \otimes \overline{\mathbf{5}}_{j} \otimes \overline{\mathbf{5}}_{H_{d}}\right)_{a b},
$$

with $i, j$ denoting the family index. Relevant calculations can be found in our previous works [37-40]. Similar calculations give the resulting trilinear terms

$$
\begin{aligned}
-\mathcal{L} & \supset \frac{F_{75}}{M_{*}\left(Z_{U}\right)^{3 / 2}}\left(1+c_{1} \frac{\langle S\rangle}{M_{*}}\right)\left(3 y_{i j}^{E} \tilde{L}_{i} \tilde{E}_{j} H_{d}-y_{i j}^{D} \tilde{Q}^{i} \tilde{D}_{j} H_{d}\right) \\
& \approx \frac{F_{75}}{M_{*}\left(Z_{U}\right)^{1 / 2}}\left(3 y_{i j}^{E} \tilde{L}_{i} \tilde{E}_{j} H_{d}-y_{i j}^{D} \tilde{Q}^{i} \tilde{D}_{j} H_{d}\right) .
\end{aligned}
$$

after rescaling the kinetic factor $Z_{U}$ for sfermions and higgs chiral fields $\mathbf{5}_{H_{u}}, \overline{\mathbf{5}}_{H_{d}}$. Note that our previous calculations [37-40] indicate that the up-type squark trilinear terms vanish if we only introduce the F-term VEV for $\mathbf{7 5}$. 
The SUSY preserving $\mu$ term, which will be determined by the electroweak symmetry breaking conditions, is generated by fine tuning with the lowest component VEV of $\Phi^{\mathbf{2 4}}$

$$
W \supset\left(M+\left\langle\Phi^{24}\right\rangle\right) \mathbf{5}_{H_{u}} \overline{\mathbf{5}}_{H_{d}} .
$$

Because one cannot construct gauge invariant combinations involving only $\mathbf{5}, \overline{\mathbf{5}}$ and $\mathbf{7 5}$, the $B_{\mu}$ term can be generated from

$$
W \supset \frac{1}{M_{*}^{2}}\left(M+\left\langle\Phi^{24}\right\rangle\right) \Phi^{24} \Phi^{75} \boldsymbol{5}_{H_{u}} \overline{\mathbf{5}}_{H_{d}}
$$

which gives

$$
B_{\mu}=\frac{5}{6 \sqrt{3}} \frac{v_{24}}{M_{*}} \frac{F_{75}}{M_{*}} \mu .
$$

So we can see that the $B_{0} \equiv B_{\mu} / \mu$ term at the GUT scale is suppressed by a GUT/Planck factor relative to $A_{0}$ and can be set to zero at the GUT scale.

We can introduce only the $\mathbf{2 4}$ or $\mathbf{2 0 0}$ representation field as the GUT non-singlet field $\Phi$ in the generalized gauge kinetic terms and then the GUT scale non-universal gaugino $[61,62]$ input will be changed accordingly:

- The scenario with only $\mathbf{2 4}$ representation Higgs:

The lowest component VEV for the $\mathbf{2 4}$ representation field has the form

$$
<\Phi_{\mathbf{2 4}}>_{a b}=v_{\mathbf{2 4}} U_{a b},
$$

with the universal group factor $U_{a b}$ given in terms of $5 \times 5$ matrix by

$$
U_{a b}=\frac{1}{\sqrt{15}}\left(1,1,1,-\frac{3}{2},-\frac{3}{2}\right) .
$$

Similar to the case of $\mathbf{7 5}$ representation Higgs, the ratios of the non-universal parts within the wave-function normalization factor of gauge kinetic terms will be given by $\beta_{1}: \beta_{2}: \beta_{3}=1: 3:-2$. This will lead to GUT scale non-universal gaugino input:

$$
M_{1}: M_{2}: M_{3}=\frac{1}{3}: \frac{3}{5}: \frac{-2}{Z_{3}} \simeq \mathcal{O}(10) .
$$

- The scenario with only $\mathbf{2 0 0}$ representation Higgs:

The lowest component VEV for the $\mathbf{2 0 0}$ representation field has the form

$$
<\Phi_{200}>_{a b}=v_{200} U_{a b},
$$

with the universal group factor $U_{a b}$ given in terms of $15 \times 15$ matrix by

$$
U_{a b}=\frac{1}{\sqrt{12}}(\underbrace{1, \cdots, 1}_{6}, \underbrace{-2, \cdots,-2}_{6}, \underbrace{2, \cdots, 2}_{3}) .
$$

The ratios of the non-universal parts within the wave-function normalization factor of gauge kinetic terms are given by $\beta_{1}: \beta_{2}: \beta_{3}=10: 2: 1$. This will lead to a GUT scale non-universal gaugino input:

$$
M_{1}: M_{2}: M_{3}=\frac{10}{9}: \frac{2}{1}: \frac{1}{Z_{3}} \simeq \mathcal{O}(10) .
$$


So we see that such a non-universal gaugino scenario with very heavy gluino at the GUT scale can be naturally obtained with proper high dimensional operators in the framework of SU(5) GUT.

\section{Phenomenological constraints}

Now we scan the parameter space of our gluino-SUGRA scenario. The GUT scale inputs can are given by

- The gaugino mass scale $M_{1 / 2}$ with non-universal gaugino mass raito

$$
M_{1}: M_{2}: M_{3}=\frac{5}{6}: \frac{3}{2}: \frac{1}{Z_{3}}(\sim \mathcal{O}(10)),
$$

for the case with the $\mathbf{7 5}$ representation Higgs. Here we define $M_{1 / 2}$ as $M_{1}=$ $(5 / 6) M_{1 / 2}$ and in our numerical calculations we will vary $1 / Z_{3}$ from 10 to 50 . At the weak scale, the gaugino mass ratio is estimated to be

$$
M_{1}: M_{2}: M_{3} \approx \frac{5}{6}: 3: \frac{6}{Z_{3}}
$$

- The universal sfermion mass $M_{S}$.

- The trilinear term $A_{b, \tau}$ (at the same order as $M_{S}$ ) while $A_{t}=0$.

- The $B_{0}$ parameter is set to zero at the GUT scale.

- The parameter $\tan \beta$ (in our scan we vary it in the range $1<\tan \beta<50$ ). Choices of $\tan \beta$ which can not trigger successful radiative EWSB will not be kept in our numerical scan.

The $\mu$ parameter is determined by the electroweak symmetry breaking conditions.

We use the code DarkSUSY [64] to scan over the parameter space and use the code SuSpect2 [63] to obtain the low energy spectrum by RGE running from the GUT scale (at this energy scale $g_{1}=g_{2}$ ) to the weak scale. The central values of $g_{1}, g_{2}$ and $g_{3}$ at the weak scale are used as the inputs. Other inputs, for example, the top Yukawa coupling $h_{t}$, are extracted from the standard model taking into account the threshold corrections (the relevant details can be seen in the appendix of [65-67]).

In our scan we consider the following constraints (the relevant details can be found in our previous work [68]):

(1) The relic density of the neutralino dark matter given by Planck $\Omega_{D M}=0.1199 \pm$ 0.0027 [69] (in combination with the WMAP data [70]).

(2) The LEP lower bounds on neutralinos and charginos $\left(m_{\chi^{C}}>103 \mathrm{GeV}\right)$ as well as the bounds from invisible $Z$ decay $\Gamma\left(Z \rightarrow \chi^{0} \chi^{0}\right)<1.71 \mathrm{MeV}$ which is consistent with the $2 \sigma$ precision EW measurement result $\Gamma_{\text {inv }}^{\text {non- }}$ SM $<2.0 \mathrm{MeV}$. 
(3) The precison electroweak observables $S, T, U[71,72]$ to be compatible with the LEP/SLD data at $2 \sigma$ level [73].

(4) The LHC constraints on the SM-like Higgs boson mass $123 \mathrm{GeV}<M_{h}<127 \mathrm{GeV}[1,2]$.

In our scan, we also require that the survived points should satisfy successful EW symmetry breaking requirements which otherwise will not be kept. Under the above constraints we will show the SUSY contributions to the muon $g_{\mu}-2$ and the spin-independent dark matter-nucleon scattering rates compared with the dark matter direct detection limits from Xenon100 [74] and the LUX [75]:

- For the spin-independent dark matter-nucleon scattering rate, we calculate it with the parameters [76-79]: $f_{T_{u}}^{(p)}=0.023, f_{T_{d}}^{(p)}=0.032, f_{T_{u}}^{(n)}=0.017, f_{T_{d}}^{(n)}=0.041$ and $f_{T_{s}}^{(p)}=$ $f_{T_{s}}^{(n)}=0.020$. The value of $f_{T_{s}}$ is taken from the lattice simulation results [80-82]. All the contributions known so far, including QCD corrections, are taken into account in our calculation of the scattering rate.

- For the SUSY contributions to the muon $g_{\mu}-2$, we know that they are dominated by the chargino-sneutrino and neutralino-smuon loops. At the leading order of $m_{W} / m_{\mathrm{SUSY}}$ and $\tan \beta$ ( $m_{\mathrm{SUSY}}$ denotes the SUSY-breaking masses), the SUSY loop contributions are [83-88]

$$
\begin{aligned}
& \Delta a_{\mu}\left(\tilde{W}, \tilde{H}, \tilde{\nu}_{\mu}\right) \simeq 15 \times 10^{-9}\left(\frac{\tan \beta}{10}\right)\left(\frac{(100 \mathrm{GeV})^{2}}{\mu M_{2}}\right) \\
& \Delta a_{\mu}\left(\tilde{W}, \tilde{H}, \tilde{\mu}_{L}\right) \simeq-2.5 \times 10^{-9}\left(\frac{\tan \beta}{10}\right)\left(\frac{(100 \mathrm{GeV})^{2}}{\mu M_{2}}\right) \\
& \Delta a_{\mu}\left(\tilde{B}, \tilde{H}, \tilde{\mu}_{L}\right) \simeq 0.76 \times 10^{-9}\left(\frac{\tan \beta}{10}\right)\left(\frac{(100 \mathrm{GeV})^{2}}{\mu M_{1}}\right) \\
& \Delta a_{\mu}\left(\tilde{B}, \tilde{H}, \tilde{\mu}_{R}\right) \simeq-1.5 \times 10^{-9}\left(\frac{\tan \beta}{10}\right)\left(\frac{(100 \mathrm{GeV})^{2}}{\mu M_{1}}\right) \\
& \Delta a_{\mu}\left(\tilde{\mu}_{L}, \tilde{\mu}_{R}, \tilde{B}\right) \simeq 1.5 \times 10^{-9}\left(\frac{\tan \beta}{10}\right)\left(\frac{(100 \mathrm{GeV})^{2}\left(\mu M_{1}\right)}{m_{\tilde{\mu}_{L}}^{2} m_{\tilde{\mu}_{R}}^{2}}\right),
\end{aligned}
$$

The SUSY contributions to the muon $g_{\mu}-2$ will be enhanced for small soft masses and large $\tan \beta$. Since the experimental value is larger than the SM prediction, a positive $\mu M_{1,2}$ is favored in most of the parameter space. See also the results from the numerical code [89].

The numerical results from our scan are shown in figure 1, figure 2, figure 3 and table 1 . All the points in the figures can satisfy the constraints (1-4), where the green ' $\square$ ' (red ' $\triangle^{\prime}$ ) can (cannot) explain the muon $g_{\mu}-2$ deviation $\Delta a_{\mu}=(255 \pm 80) \times 10^{-11}$ at $1 \sigma$ level. As shown in these figures, some samples in our gluino-SUGRA scenario can satisfy the LHC constraints and explain the Brookhaven $g_{\mu}-2$ experiment. For these samples, we have the following observations: 

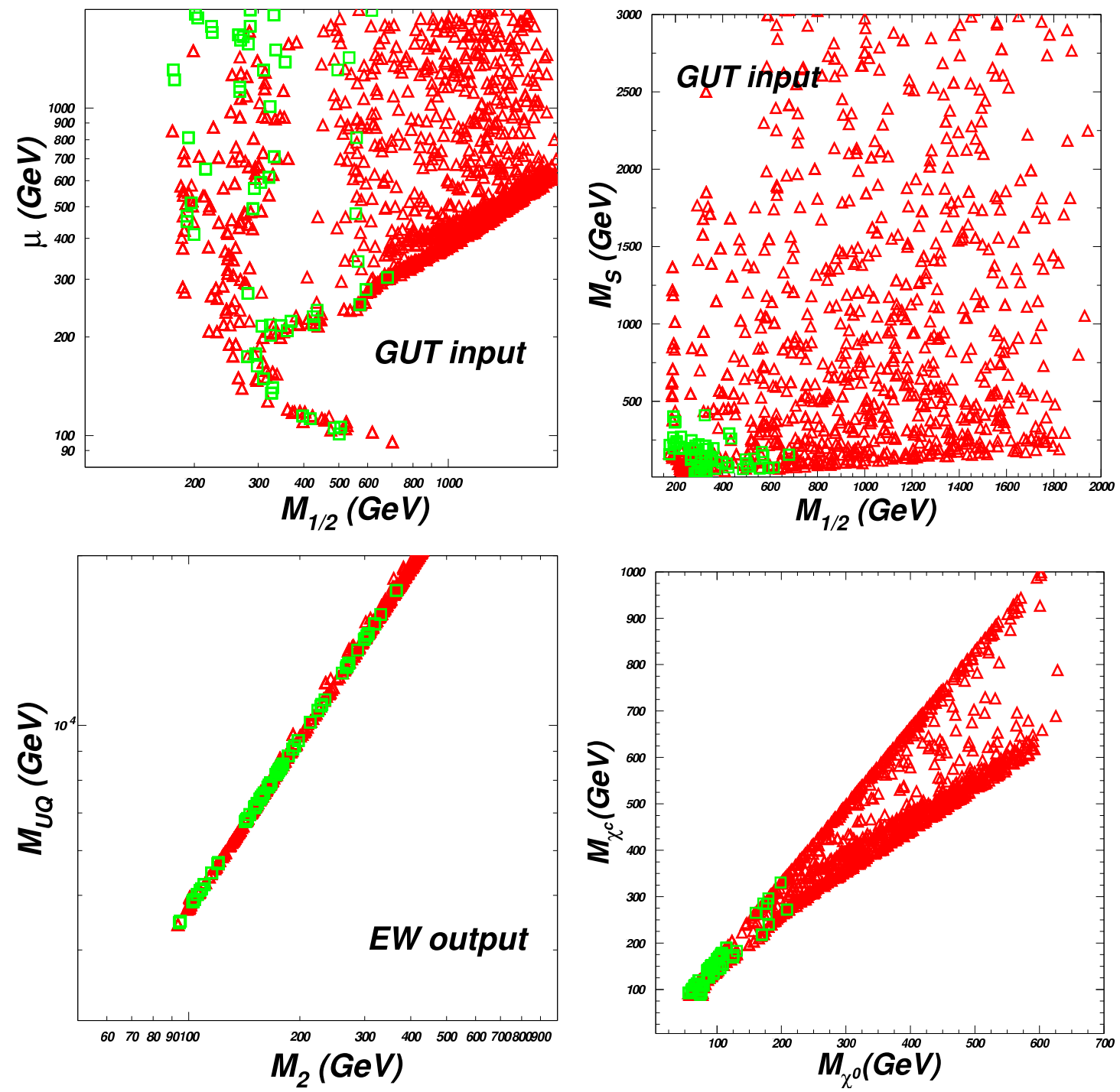

Figure 1. The scatter plots of the samples that satisfy the constraints (1-4) for $k \equiv 1 / Z_{3}=10$. The green ' $\square^{\prime}\left(\right.$ red $\left.{ }^{\prime} \triangle^{\prime}\right)$ can (cannot) explain the muon $g_{\mu}-2$ at $1 \sigma$ level. The upper panels show the input parameters at GUT scale while the lower panels show the output parameters at electroweak (EW) scale, with $M_{U Q}$ denoting the up-squark soft mass, $M_{\chi^{C}}$ the lightest chargino mass and $M_{\chi^{0}}$ the lightest neutralino mass.

(i) From the upper left panel of figure 1, we can see that the muon $g_{\mu}-2$ explanation constrains $M_{1 / 2}$ (defined as $M_{1}=(5 / 6) M_{1 / 2}$ at the GUT scale) below $600 \mathrm{GeV}$. The upper right panel shows the muon $g_{\mu}-2$ explanation also requires a light value for the universal sfermion mass $M_{S}$ at the GUT scale (so that at the electroweak scale we have light sleptons and electroweakinos while squarks are heavy due to RGE running). Such results can be easily understood from eqs. (3.3)-(3.7).

(ii) Due to a rather heavy gluino, when squark masses run down to the electroweak scale, they become sufficiently heavy (although $M_{S}$ is light at the GUT scale) as required 


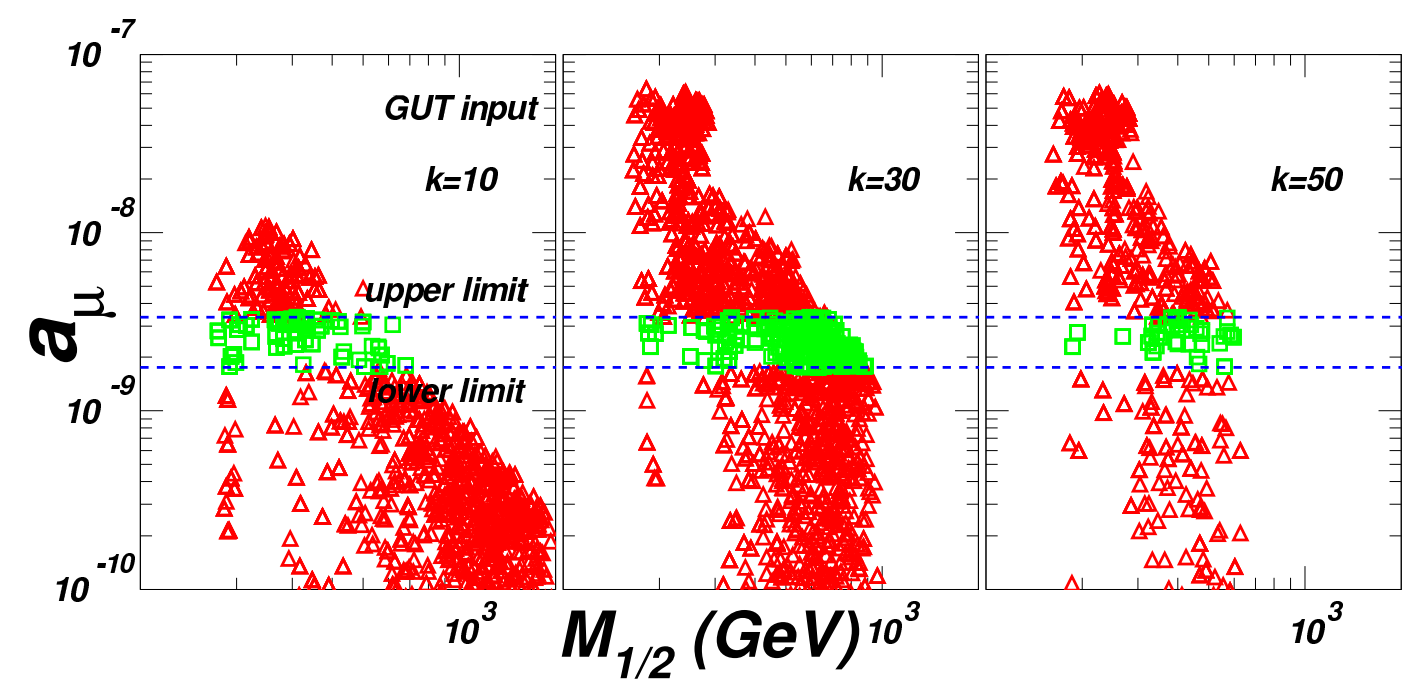

Figure 2. Same as figure 1 , but showing the muon $g_{\mu}-2$ versus $M_{1 / 2}$ for different values of $k \equiv 1 / Z_{3}$. The region between the two horizontal dashed lines corresponds to the Brookhaven measured $g_{\mu}-2$ at $1 \sigma$ level.

\begin{tabular}{|ccccccccc|}
\hline$k$ & $M_{1 / 2}$ & $\mu$ & $m_{\chi^{0}}$ & $m_{\tilde{g}}$ & $m_{\tilde{\mu}_{1}}$ & $m_{\tilde{\mu}_{2}}$ & $m_{\tilde{u}_{1}}$ & $m_{\tilde{d}_{1}}$ \\
\hline 10 & 297.63 & 163.09 & 79.54 & 8583.95 & 273.28 & 246.47 & 7594.43 & 7594.35 \\
\hline 30 & 535.07 & 367.18 & 190.49 & 46289.43 & 195.09 & 286.52 & 40934.25 & 40934.15 \\
\hline 50 & 398.92 & 213.81 & 132.09 & 57522.28 & 171.78 & 232.77 & 50867.91 & 50867.88 \\
\hline
\end{tabular}

Table 1. The masses (in GeV) of some sparticles at the weak scale for different values of $k \equiv 1 / Z_{3}$. All the points satisfy $g_{\mu}-2$ constraints and other electroweak constraints.

by a $125 \mathrm{GeV}$ SM-like Higgs boson and the LHC bounds. We can see from the lower left panel of figure 1 that the squarks at the electroweak scale are heavier than $4 \mathrm{TeV}$ in our scenario.

(iii) From the lower right panel of figure 1 we see that the muon $g_{\mu}-2$ explanation requires low masses for the lightest chargino and the lightest neutralino. The lightest neutralino dark matter lies in the mass range of 80 to $200 \mathrm{GeV}$.

(iv) From figure 3 we see that for $k=10(k \geq 30)$ most (all) samples required to explain the muon $g_{\mu}-2$ at $1 \sigma$ level can be covered by the future Xenon1T experiment. This means that in case of null results at Xenon1T experiment, our scenario with $k \geq 30$ will be excluded.

(v) The low energy particle spectrum for some typical benchmark points are shown in table 1 . We can see that the sleptons are typically light while the squarks are heavy due to the much heavier gluino. 

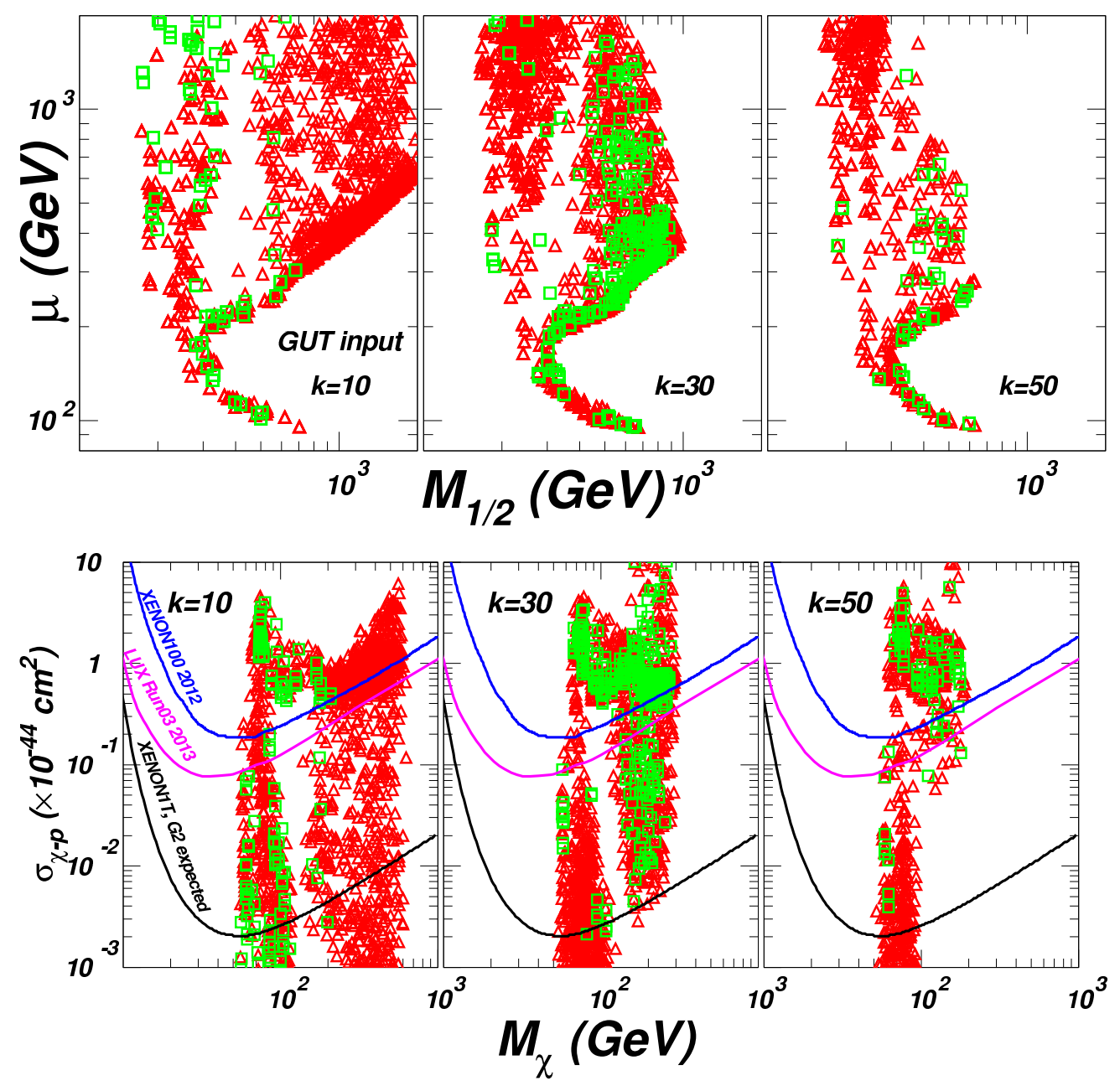

Figure 3. Same as figure 2, but showing $\mu$ versus $M_{1 / 2}$ in the upper panel and the spin-independent dark matter-nucleon scattering rate versus the lightest neutralino dark matter mass in the lower panel. The curves in the lower panel show the dark matter direct detection limits from Xenon [74] and the LUX [75].

\section{Conclusion}

From generalized gravity mediation we constructed a SUGRA scenario in which the gluino is much heavier than the electroweak gauginos at the GUT scale. We chose the framework of SU(5) GUT and found that such a non-universal gaugino scenario with very heavy gluino at the GUT scale can be naturally obtained with proper high dimensional operators. Due to the contributions of the heavy gluino, at the weak scale the squarks are sufficiently heavy as required by a $125 \mathrm{GeV}$ SM-like Higgs boson, while the uncolored spartilces can be light enough to explain the Brookhaven muon $g-2$ measurement. Since the muon $g-2$ explanation requires a neutralino dark matter below $200 \mathrm{GeV}$ in our scenario, the parameter space can be mostly covered by the future Xenon1T experiment. 


\section{Acknowledgments}

This work was supported by the Natural Science Foundation of China under grant numbers 11105124, 11105125, 11275245, 10821504, 11135003, 11375001, 11172008, by Ri-Xin Foundation of BJUT and by tanlents foundation of eduction department of Beijing.

Open Access. This article is distributed under the terms of the Creative Commons Attribution License (CC-BY 4.0), which permits any use, distribution and reproduction in any medium, provided the original author(s) and source are credited.

\section{References}

[1] ATLAS collaboration, Combined search for the Standard Model Higgs boson using up to $4.9 \mathrm{fb}^{-1}$ of pp collision data at $\sqrt{s}=7 \mathrm{TeV}$ with the ATLAS detector at the LHC, Phys. Lett. B 710 (2012) 49 [arXiv:1202.1408] [INSPIRE].

[2] CMS collaboration, Combined results of searches for the standard model Higgs boson in pp collisions at $\sqrt{s}=7 \mathrm{TeV}$, Phys. Lett. B 710 (2012) 26 [arXiv:1202.1488] [INSPIRE].

[3] Muon G-2 collaboration, G.W. Bennett et al., Final Report of the Muon E821 Anomalous Magnetic Moment Measurement at BNL, Phys. Rev. D 73 (2006) 072003 [hep-ex/0602035] [INSPIRE].

[4] B.L. Roberts, Status of the Fermilab Muon (g-2) Experiment, Chin. Phys. C 34 (2010) 741 [arXiv: 1001.2898].

[5] K. Hagiwara, A.D. Martin, D. Nomura and T. Teubner, Improved predictions for g-2 of the muon and $\alpha_{\mathrm{QED}}\left(M_{Z}^{2}\right)$, Phys. Lett. B 649 (2007) 173 [hep-ph/0611102] [INSPIRE].

[6] T. Teubner, K. Hagiwara, R. Liao, A. Martin and D. Nomura, Update of g-2 of the muon and Delta alpha, Chin. Phys. C 34 (2010) 728 [arXiv:1001.5401].

[7] M. Davier et al., The Discrepancy Between tau and $e^{+} e^{-}$Spectral Functions Revisited and the Consequences for the Muon Magnetic Anomaly, Eur. Phys. J. C 66 (2010) 127 [arXiv: 0906.5443] [INSPIRE].

[8] M. Davier, A. Hoecker, B. Malaescu, C.Z. Yuan and Z. Zhang, Reevaluation of the hadronic contribution to the muon magnetic anomaly using new $e^{+} e^{-} \rightarrow \pi^{+} \pi^{-}$cross section data from BABAR, Eur. Phys. J. C 66 (2010) 1 [arXiv:0908.4300] [INSPIRE].

[9] H. Georgi and S.L. Glashow, Unity of All Elementary Particle Forces, Phys. Rev. Lett. 32 (1974) 438 [INSPIRE].

[10] J.R. Ellis, S. Kelley and D.V. Nanopoulos, Precision LEP data, supersymmetric GUTs and string unification, Phys. Lett. B 249 (1990) 441 [INSPIRE].

[11] U. Amaldi, W. de Boer and H. Furstenau, Comparison of grand unified theories with electroweak and strong coupling constants measured at LEP, Phys. Lett. B 260 (1991) 447 [INSPIRE].

[12] P. Langacker and M.-x. Luo, Implications of precision electroweak experiments for $M_{t}, \rho_{0}$, $\sin ^{2} \theta_{W}$ and grand unification, Phys. Rev. D 44 (1991) 817 [INSPIRE].

[13] M.B. Einhorn and D.R.T. Jones, The Weak Mixing Angle and Unification Mass in Supersymmetric SU(5), Nucl. Phys. B 196 (1982) 475 [inSPIRE]. 
[14] W.J. Marciano and G. Senjanović, Predictions of Supersymmetric Grand Unified Theories, Phys. Rev. D 25 (1982) 3092 [inSPIRE].

[15] J.-J. Cao, Z.-X. Heng, J.M. Yang, Y.-M. Zhang and J.-Y. Zhu, A SM-like Higgs near $125 \mathrm{GeV}$ in low energy SUSY: a comparative study for MSSM and NMSSM, JHEP 03 (2012) 086 [arXiv: 1202.5821] [INSPIRE].

[16] J. Cao, Z. Heng, J.M. Yang and J. Zhu, Status of low energy SUSY models confronted with the LHC $125 \mathrm{GeV}$ Higgs data, JHEP 10 (2012) 079 [arXiv:1207.3698] [INSPIRE].

[17] ATLAS collaboration, Search for squarks and gluinos using final states with jets and missing transverse momentum with the ATLAS detector in $\sqrt{s}=7 \mathrm{TeV}$ proton-proton collisions, Phys. Lett. B 710 (2012) 67 [arXiv:1109.6572] [INSPIRE].

[18] ATLAS collaboration, Search for squarks and gluinos with the ATLAS detector in final states with jets and missing transverse momentum using $4.7 \mathrm{fb}^{-1}$ of $\sqrt{\mathrm{s}}=7 \mathrm{TeV}$ proton-proton collision data, Phys. Rev. D 87 (2013) 012008 [arXiv:1208.0949] [INSPIRE].

[19] CMS collaboration, Search for Supersymmetry at the LHC in Events with Jets and Missing Transverse Energy, Phys. Rev. Lett. 107 (2011) 221804 [arXiv:1109.2352] [InSPIRE].

[20] CMS collaboration, Search for supersymmetry in hadronic final states using MT2 in pp collisions at $\sqrt{s}=7 \mathrm{TeV}$, JHEP 10 (2012) 018 [arXiv: 1207.1798] [INSPIRE].

[21] C. Han, K.-i. Hikasa, L. Wu, J.M. Yang and Y. Zhang, Current experimental bounds on stop mass in natural SUSY, JHEP 10 (2013) 216 [arXiv:1308.5307] [INSPIRE].

[22] J. Chakrabortty, A. Choudhury and S. Mondal, Non-universal Gaugino mass models under the lamppost of muon $(g-2)$, arXiv: 1503.08703 [INSPIRE].

[23] K. Kowalska, L. Roszkowski, E.M. Sessolo and A.J. Williams, GUT-inspired SUSY and the muon g-2 anomaly: prospects for LHC 14 TeV, JHEP 06 (2015) 020 [arXiv: 1503.08219] [INSPIRE].

[24] K. Harigaya, T.T. Yanagida and N. Yokozaki, Higgs boson mass of $125 \mathrm{GeV}$ and $g-2$ of the muon in a gaugino mediation model, Phys. Rev. D 91 (2015) 075010 [arXiv:1501.07447] [INSPIRE].

[25] M. Adeel Ajaib, I. Gogoladze and Q. Shafi, GUT-inspired supersymmetric model for $h \rightarrow \gamma \gamma$ and the muon $g$ - 2, Phys. Rev. D 91 (2015) 095005 [arXiv:1501.04125] [InSPIRE].

[26] F.F. Deppisch, N. Desai and T.E. Gonzalo, Compressed and Split Spectra in Minimal SUSY SO(10), Front. Phys. 2 (2014) 00027 [arXiv:1403.2312] [InSPIRE].

[27] T. Cheng, J. Li, T. Li, D.V. Nanopoulos and C. Tong, Electroweak Supersymmetry around the Electroweak Scale, Eur. Phys. J. C 73 (2013) 2322 [arXiv:1202.6088] [INSPIRE].

[28] T. Li and S. Raza, Electroweak supersymmetry from the generalized minimal supergravity model in the MSSM, Phys. Rev. D 91 (2015) 055016 [arXiv:1409.3930] [INSPIRE].

[29] S. Akula and P. Nath, Gluino-driven radiative breaking, Higgs boson mass, muon $g-2$ and the Higgs diphoton decay in supergravity unification, Phys. Rev. D 87 (2013) 115022 [arXiv:1304.5526] [INSPIRE].

[30] N. Chamoun, C.-S. Huang, C. Liu and X.-H. Wu, Nonuniversal gaugino masses in supersymmetric SO(10), Nucl. Phys. B 624 (2002) 81 [hep-ph/0110332] [INSPIRE]. 
[31] K. Huitu, J. Laamanen, P.N. Pandita and S. Roy, Phenomenology of non-universal gaugino masses in supersymmetric grand unified theories, Phys. Rev. D 72 (2005) 055013 [hep-ph/0502100] [INSPIRE].

[32] K. Huitu et al., Search for Higgs Bosons in SUSY Cascades in CMS and Dark Matter with Non-universal Gaugino Masses, Eur. Phys. J. C 58 (2008) 591 [arXiv:0808.3094] [INSPIRE].

[33] J. Chakrabortty and A. Raychaudhuri, A Note on dimension-5 operators in GUTs and their impact, Phys. Lett. B 673 (2009) 57 [arXiv:0812.2783] [INSPIRE].

[34] S.P. Martin, Non-universal gaugino masses from non-singlet F-terms in non-minimal unified models, Phys. Rev. D 79 (2009) 095019 [arXiv:0903.3568] [INSPIRE].

[35] D. Horton and G.G. Ross, Naturalness and Focus Points with Non-Universal Gaugino Masses, Nucl. Phys. B 830 (2010) 221 [arXiv:0908.0857] [INSPIRE].

[36] F.F. Deppisch, N. Desai and T.E. Gonzalo, Compressed and Split Spectra in Minimal SUSY SO(10), Front. Phys. 2 (2014) 00027 [arXiv: 1403.2312].

[37] C. Balázs, T. Li, D.V. Nanopoulos and F. Wang, Supersymmetry Breaking Scalar Masses and Trilinear Soft Terms in Generalized Minimal Supergravity, JHEP 09 (2010) 003 [arXiv: 1006.5559] [INSPIRE].

[38] T. Li and D.V. Nanopoulos, Generalizing Minimal Supergravity, Phys. Lett. B 692 (2010) 121 [arXiv:1002.4183] [INSPIRE].

[39] C. Balázs, T. Li, D.V. Nanopoulos and F. Wang, Realistic Standard Model Fermion Mass Relations in Generalized Minimal Supergravity (GmSUGRA), JHEP 02 (2011) 096 [arXiv:1101.5423] [INSPIRE].

[40] F. Wang, Supersymmetry Breaking Scalar Masses and Trilinear Soft Terms From High-Dimensional Operators in $E_{6}$ SUSY GUT, Nucl. Phys. B 851 (2011) 104 [arXiv: 1103.0069] [INSPIRE].

[41] A.H. Chamseddine, R.L. Arnowitt and P. Nath, Locally Supersymmetric Grand Unification, Phys. Rev. Lett. 49 (1982) 970 [INSPIRE].

[42] H.P. Nilles, Dynamically Broken Supergravity and the Hierarchy Problem, Phys. Lett. B 115 (1982) 193 [inSPIRE].

[43] L.E. Ibáñez, Locally Supersymmetric SU(5) Grand Unification, Phys. Lett. B 118 (1982) 73 [INSPIRE].

[44] R. Barbieri, S. Ferrara and C.A. Savoy, Gauge Models with Spontaneously Broken Local Supersymmetry, Phys. Lett. B 119 (1982) 343 [INSPIRE].

[45] H.P. Nilles, M. Srednicki and D. Wyler, Weak Interaction Breakdown Induced by Supergravity, Phys. Lett. B 120 (1983) 346 [InSPIRE].

[46] J.R. Ellis, D.V. Nanopoulos and K. Tamvakis, Grand Unification in Simple Supergravity, Phys. Lett. B 121 (1983) 123 [INSPIRE].

[47] J.R. Ellis, J.S. Hagelin, D.V. Nanopoulos and K. Tamvakis, Weak Symmetry Breaking by Radiative Corrections in Broken Supergravity, Phys. Lett. B 125 (1983) 275 [InSPIRE].

[48] N. Ohta, Grand unified theories based on local supersymmetry, Prog. Theor. Phys. 70 (1983) 542 [InSPIRE]. 
[49] L.J. Hall, J.D. Lykken and S. Weinberg, Supergravity as the Messenger of Supersymmetry Breaking, Phys. Rev. D 27 (1983) 2359 [INSPIRE].

[50] M. Dine, W. Fischler and M. Srednicki, Supersymmetric Technicolor, Nucl. Phys. B 189 (1981) 575 [inSPIRE].

[51] S. Dimopoulos and S. Raby, Supercolor, Nucl. Phys. B 192 (1981) 353 [inSPIRE].

[52] M. Dine and W. Fischler, A Phenomenological Model of Particle Physics Based on Supersymmetry, Phys. Lett. B 110 (1982) 227 [INSPIRE].

[53] M. Dine and A.E. Nelson, Dynamical supersymmetry breaking at low-energies, Phys. Rev. D 48 (1993) 1277 [hep-ph/9303230] [INSPIRE].

[54] M. Dine, A.E. Nelson and Y. Shirman, Low-energy dynamical supersymmetry breaking simplified, Phys. Rev. D 51 (1995) 1362 [hep-ph/9408384] [INSPIRE].

[55] M. Dine, A.E. Nelson, Y. Nir and Y. Shirman, New tools for low-energy dynamical supersymmetry breaking, Phys. Rev. D 53 (1996) 2658 [hep-ph/9507378] [INSPIRE].

[56] G.F. Giudice and R. Rattazzi, Theories with gauge mediated supersymmetry breaking, Phys. Rept. 322 (1999) 419 [hep-ph/9801271] [INSPIRE].

[57] L. Randall and R. Sundrum, Out of this world supersymmetry breaking, Nucl. Phys. B 557 (1999) 79 [hep-th/9810155] [INSPIRE].

[58] G.F. Giudice, M.A. Luty, H. Murayama and R. Rattazzi, Gaugino mass without singlets, JHEP 12 (1998) 027 [hep-ph/9810442] [INSPIRE].

[59] S. Akula, B. Altunkaynak, D. Feldman, P. Nath and G. Peim, Higgs Boson Mass Predictions in SUGRA Unification, Recent LHC-7 Results and Dark Matter, Phys. Rev. D 85 (2012) 075001 [arXiv:1112.3645] [INSPIRE].

[60] S. Akula, P. Nath and G. Peim, Implications of the Higgs Boson Discovery for mSUGRA, Phys. Lett. B 717 (2012) 188 [arXiv:1207.1839] [INSPIRE].

[61] J.R. Ellis, K. Enqvist, D.V. Nanopoulos and K. Tamvakis, Gaugino Masses and Grand Unification, Phys. Lett. B 155 (1985) 381 [INSPIRE].

[62] M. Drees, Phenomenological Consequences of $N=1$ Supergravity Theories With Nonminimal Kinetic Energy Terms for Vector Superfields, Phys. Lett. B 158 (1985) 409 [InSPIRE].

[63] A. Djouadi, J.-L. Kneur and G. Moultaka, SuSpect: A Fortran code for the supersymmetric and Higgs particle spectrum in the MSSM, Comput. Phys. Commun. 176 (2007) 426 [hep-ph/0211331] [INSPIRE].

[64] P. Gondolo et al., DarkSUSY: Computing supersymmetric dark matter properties numerically, JCAP 07 (2004) 008 [astro-ph/0406204] [INSPIRE].

[65] F. Wang, W. Wang and J.M. Yang, Split supersymmetry under GUT and current dark matter constraints, Eur. Phys. J. C 74 (2014) 3121 [arXiv:1310.1750] [INSPIRE].

[66] N. Bernal, A. Djouadi and P. Slavich, The MSSM with heavy scalars, JHEP 07 (2007) 016 [arXiv: 0705.1496] [INSPIRE].

[67] N. Bernal, Dark matter direct detection in the MSSM with heavy scalars, JCAP 08 (2009) 022 [arXiv: 0905.4239] [INSPIRE].

[68] F. Wang, W. Wang and J.M. Yang, A split SUSY model from SUSY GUT, JHEP 03 (2015) 050 [arXiv: 1501.02906] [INSPIRE]. 
[69] http://www.sciops.esa.int/SA/PLANCK/docs/Planck 2013 results 16.pdf.

[70] WMAP collaboration, J. Dunkley et al., Five-Year Wilkinson Microwave Anisotropy Probe (WMAP) Observations: Likelihoods and Parameters from the WMAP data, Astrophys. J. Suppl. 180 (2009) 306 [arXiv:0803.0586] [INSPIRE].

[71] G. Altarelli and R. Barbieri, Vacuum polarization effects of new physics on electroweak processes, Phys. Lett. B 253 (1991) 161 [INSPIRE].

[72] M.E. Peskin and T. Takeuchi, Estimation of oblique electroweak corrections, Phys. Rev. D 46 (1992) 381 [InSPIRE].

[73] AlePh, DelPhi, L3, OPAL, SLD, LeP Electroweak Working Group, SLD Electroweak Group and SLD Heavy Flavour Group collaborations, Precision electroweak measurements on the $Z$ resonance, Phys. Rept. 427 (2006) 257 [hep-ex/0509008] [INSPIRE].

[74] XENON100 collaboration, E. Aprile et al., Dark Matter Results from 225 Live Days of XENON100 Data, Phys. Rev. Lett. 109 (2012) 181301 [arXiv:1207.5988] [INSPIRE].

[75] LUX collaboration, D.S. Akerib et al., First results from the LUX dark matter experiment at the Sanford Underground Research Facility, Phys. Rev. Lett. 112 (2014) 091303 [arXiv:1310.8214] [INSPIRE].

[76] A. Djouadi and M. Drees, QCD corrections to neutralino nucleon scattering, Phys. Lett. B 484 (2000) 183 [hep-ph/0004205] [INSPIRE].

[77] G. Bélanger, F. Boudjema, A. Pukhov and A. Semenov, Dark matter direct detection rate in a generic model with MicrOMEGAs 2.2, Comput. Phys. Commun. 180 (2009) 747 [arXiv:0803.2360] [INSPIRE].

[78] M. Carena, D. Garcia, U. Nierste and C.E.M. Wagner, Effective Lagrangian for the $\bar{t} b H^{+}$ interaction in the MSSM and charged Higgs phenomenology, Nucl. Phys. B 577 (2000) 88 [hep-ph/9912516] [INSPIRE].

[79] J. Hisano, K. Ishiwata and N. Nagata, Gluon contribution to the dark matter direct detection, Phys. Rev. D 82 (2010) 115007 [arXiv:1007.2601] [InSPIRE].

[80] H. Ohki et al., Nucleon sigma term and strange quark content from lattice QCD with exact chiral symmetry, Phys. Rev. D 78 (2008) 054502 [arXiv:0806.4744] [INSPIRE].

[81] MILC collaboration, D. Toussaint and W. Freeman, The Strange quark condensate in the nucleon in $2+1$ flavor QCD, Phys. Rev. Lett. 103 (2009) 122002 [arXiv:0905.2432] [INSPIRE].

[82] J. Giedt, A.W. Thomas and R.D. Young, Dark matter, the CMSSM and lattice QCD, Phys. Rev. Lett. 103 (2009) 201802 [arXiv:0907.4177] [INSPIRE].

[83] T. Moroi, The Muon anomalous magnetic dipole moment in the minimal supersymmetric standard model, Phys. Rev. D 53 (1996) 6565 [Erratum ibid. D 56 (1997) 4424] [hep-ph/9512396] [INSPIRE].

[84] M. Endo, K. Hamaguchi, S. Iwamoto and T. Yoshinaga, Muon g- 2 vs LHC in Supersymmetric Models, JHEP 01 (2014) 123 [arXiv:1303.4256] [INSPIRE].

[85] M. Badziak, Z. Lalak, M. Lewicki, M. Olechowski and S. Pokorski, Upper bounds on sparticle masses from muon $g-2$ and the Higgs mass and the complementarity of future colliders, JHEP 03 (2015) 003 [arXiv:1411.1450] [INSPIRE]. 
[86] H. Fargnoli, C. Gnendiger, S. Paßehr, D. Stöckinger and H. Stöckinger-Kim, Two-loop corrections to the muon magnetic moment from fermion/sfermion loops in the MSSM: detailed results, JHEP 02 (2014) 070 [arXiv:1311.1775] [INSPIRE].

[87] H. Fargnoli, C. Gnendiger, S. Paßehr, D. Stöckinger and H. Stöckinger-Kim, Non-decoupling two-loop corrections to $(g-2)_{\mu}$ from fermion/sfermion loops in the MSSM, Phys. Lett. B 726 (2013) 717 [arXiv:1309.0980] [INSPIRE].

[88] C. Gnendiger, D. Stöckinger and H. Stöckinger-Kim, The electroweak contributions to $(g-2)_{\mu}$ after the Higgs boson mass measurement, Phys. Rev. D 88 (2013) 053005 [arXiv: 1306.5546] [INSPIRE].

[89] F.S. Queiroz and W. Shepherd, New Physics Contributions to the Muon Anomalous Magnetic Moment: A Numerical Code, Phys. Rev. D 89 (2014) 095024 [arXiv:1403.2309] [InSPIRE]. 